\title{
Hematologic Neoplasms As Risk Factor For Severe COVID-19: A Systematic Review Protocol
}

Paula de Oliveira Montandon Hokama ( $\sim$ paula.hokama@unesp.br)

São Paulo State University (UNESP) https://orcid.org/0000-0003-3474-4422

Newton Key Hokama

São Paulo State University (UNESP)

Adriana Lúcia Mendes

São Paulo State University (UNESP)

Vania dos Santos Nunes-Nogueira

São Paulo State University (UNESP)

\section{Protocol}

Keywords: Hematologic Neoplasm, Blood Disease, Coronavirus infection, COVID-19,Background

Posted Date: November 15th, 2021

DOI: https://doi.org/10.21203/rs.3.rs-1053230/v1

License: (9) This work is licensed under a Creative Commons Attribution 4.0 International License.

Read Full License 
1 Hematologic neoplasms as risk factor for severe COVID-19: a systematic review

2 protocol

3

4

5 Paula de Oliveira Montandon Hokama* Corresponding author.

6 Clinical Patology Division, São Paulo State University (UNESP), Botucatu, SP, Brazil.

7 email: paula.hokama@unesp.br

Newton Key Hokama. Hematology Division, São Paulo State University (UNESP),

Botucatu, SP, Brazil.

email: newton.hokama@unesp.br

Adriana Lúcia Mendes. Endocrinology Division, São Paulo State University (UNESP), Botucatu, SP, Brazil.

email: adriana.mendes@unesp.br

Vania dos Santos Nunes-Nogueira. Endocrinology Division, São Paulo State University (UNESP), Botucatu, SP, Brazil.

email: vania.nunes-nogueira@unesp.br

\section{*Corresponding author address:}

Faculdade de Medicina de Botucatu -Unesp

Departamento de Clínica Médica.

Av. Prof. Mario Rubens Guimarães Montengro, s/n.

Campus de Botucatu - SP - 18918-687

Abstract

Background: Patients with hematologic neoplasm may have compromised immunity due to their malignancy and/or treatment, and may be at elevated risk of severe COVID-19. However, the studies bring together patients with hematologic neoplasms and solid tumors into a single group, making no distinction about the types of hematological tumors and their treatments.

This systematic review is designed to explore the risk of severe COVID-19 in patients with 
hematologic neoplasm. Studies about patients, adult or children, with hematologic neoplasm and COVID-19 will be included.

Methods: A systematic review according to Joanna Briggs Institute methodology for systematic reviews of etiology and risk will be performed. The review will consider as participants adults or children with COVID-19 infection detected by RT-PCR or serology (SARS-CoV-2 antibody). We will be included studies without routine labs confirmation of COVID-19 if the patients presented clinical/physical exam and computed tomography suggesting COVID-19. The exposure of interest will be hematologic neoplasm, which include lymphomas, acute and chronic leukemias, myeloma, myelodysplastic syndrome, and myeloproliferative diseases. We will consider cohort, case-control, analytical cross-sectional studies. Outcomes among patients with COVID-19 are critical symptoms, hospitalizations, intensive care unit admissions, mechanical ventilation and deaths. We will exclude studies with other neoplasms than hematologic neoplasms. Search strategies have been created for the Embase, Medline and LILACS. Two reviewers independently will assess the studies for their eligibility, will extract data and will evaluate their risk of bias. Similar outcomes measured in at least two studies will be plotted in the meta-analysis using the Joanna Briggs Institute System for the Unified Management, Assessment and Review of Information.

Discussion: This systematic review aims to evaluate if patients with hematologic neoplasm may be at elevated risk of severe COVID-19. This review will differ from the previous ones because we will include controlled studies and groups with only hematologic neoplasm, excluding other cancers. The main hypothesis of our research is that not all hematological cancer patients have high risk of severe COVID-19.

Trial registration number: PROSPERO CRD42020199318.

Keywords: Hematologic Neoplasm; Blood Disease; Coronavirus infection; COVID-19 Background

Hematologic neoplasms are a heterogeneous group of malignancies diseases derived from hematopoietic and lymphoid tissue. They present clinically as lymphoid or myeloid leukemia (acute or chronic), lymphoma (Hodgkin or non-Hodgkin), multiple myeloma, myelodysplastic syndrome and myeloproliferative diseases [1]. 
The natural course of hematologic neoplasms includes an immunosuppression status caused by both disease and treatment [2]. Multiple factors may contribute to decreased resistance such as neutropenia, neutrophil function defects, humoral immunity deficiency and impaired cellular immunity or a combination of these disturbances $[2,3]$.

The treatment's options for hematologic malignancies may be wait-and-watch approaches, antineoplastic agents as chemotherapy myeloablative or non-myeloablative, target therapies and immunotherapy, radiotherapy and stem cell transplantation, as well as supportive care to prevent, control or treatment complications and side effects [4]. The immunosuppressive state makes cancer patients more vulnerable to infections, increasing the risk of coronavirus infection in these patients.

A retrospective propensity score matching study involving patients with COVID-19 compared the outcomes of 109 cancer patients with 327 non-cancer controls patients. The clinical outcomes of patients with hematological malignancies were worse than patients with solid tumors [5]. A systematic review of 31 studies and meta-analysis of 181,323 patients from 26 studies involving 23,736 cancer patients showed that cancer patients with COVID19 have a higher likelihood of death. The mortality was highest in hematological malignancies followed by lung cancer [6]. However, the authors did not compare the mortality rate between cancer and individuals without cancer.

The objective of this review owns to evaluate if patients with hematologic neoplasm may be at elevated risk of severe COVID-19. Our review will differ from the previous ones because we will include controlled studies and groups with only hematologic neoplasm, excluding other cancers. The main hypothesis of our research is that not all hematological cancer patients have high risk of severe COVID-19.

The question of our protocol is if hematologic neoplasms are risk factor for severe COVID-19. Thus, for controlled studies included the comparator will be individuals with COVID-19 and without hematologic neoplasm.

A preliminary search of PROSPERO, MEDLINE, the Cochrane Database of Systematic Reviews and the Joanna Briggs Institute Database of Systematic Reviews and Implementation Reports was conducted, and we have found on the same topic two published systematic reviews and a protocol of a systematic review. The protocol and one of the published 
reviews have evaluated in patients with COVID-19 and cancer the risk of death, as well as the risk of intensive unit care admission and ventilation [7]. The second published systematic review compared outcomes of patients with hematologic malignancies and COVID-19 with patients infected with COVID-19 and without hematologic neoplasm. However, this review was published in 2020 and the authors used only PMC as source data [8]. Additionally, a nation-wide observational study in COVID-19 comparing patients with hematologic malignancies and patients without hematologic malignancies was published in 2021 [9].

Our study will address the first and second phases of the pandemic, unlike previous studies that based their results only on the first phase of the pandemic. The second phase of the pandemic was characterized by greater experience of professionals and health systems in the management and absorption of COVID-19 cases, which may have an impact on statistical analysis [7]. Another differential of our study is that we will expand the search in specific databases from countries that were the epicenter of the pandemic, such as China, Brazil, Europe and Oceania.

\section{Methods}

The proposed systematic review will be conducted in accordance with the Joanna Briggs Institute methodology for systematic reviews of etiology and risk (Chapter7: Systematic reviews of etiology and risk) [10]. The registration number of this study protocol in PROSPERO is CRD 42020199318. This protocol has being reported according to the Preferred Reporting Items for Systematic Reviews and Meta-Analysis Protocols (PRISMA-P) statement (see Additional File 1). Any amendments to the protocol will be described in the final review article.

\section{Review question}

The question of this review is 'Are hematologic neoplasms a risk factor for severe COVID-19 infection?'

\section{Eligibility Criteria}

\section{Participants}

The review will consider as participants adults ( $\geq 18$ years-old) or children ( $<18$ yearsold) with COVID-19 infection detected by RT-PCR or serology (SARS-CoV-2 antibody). We 
will be included studies without routine labs confirmation of COVID-19 if the patients presented clinical/physical exam and computed tomography suggesting COVID-19.

RT-PCR tests can be performed with nasopharyngeal, oropharyngeal, saliva, sputum, stool, blood and/or urine specimens. Clinical and physical exam can include cough, dyspnoea, sore throat or fatigue with acute smell and/or taste disorders. The changes considered in routine labs tests are lymphopenia and elevated LDH. Prothrombin Time (PT), ferritin, D-dimer or IL6 also will be included, and they are associated with severe COVID-19. Chest X-ray typical COVID-19 findings include hazy opacities that can be bilateral and peripheral. The ground glass opacity, often bilateral and peripheral is the predominant finding of COVID-19 observed on Computed Tomography of the chest. The serology assay is a SARS-CoV-2 antibody test that detects circulating $\operatorname{IgM}, \operatorname{IgG}$ or both on plasma or serum samples. Antigen tests detect SARS-Co-V or SAVS-CoV-2 nucleocapsid protein antigens in nasopharyngeal or nasal specimens [11].

We will exclude studies in which there was no laboratory confirmation of COVID-19, as well as studies with other neoplasms than hematologic neoplasm.

\section{Exposure of Interest}

The exposure of interest will be all specific hematologic neoplasm. We will consider hematologic neoplasm and all the subtypes of non-Hodgkin lymphomas, Hodgkin Disease, acute myeloid leukemia, acute lymphoid leukemia, chronic lymphoid leukemia, multiple myeloma, myelodysplastic syndrome, and myeloproliferative diseases diagnosis according to WHO criteria [12]. The exposure group will be evaluated regarding the stage of the disease based on its severity and duration and the type of treatment (wait-and-watch approaches, myeloablative or non-myeloablative chemeoterapy, target therapies and immunotherapy, radiotherapy and stem cell transplantation, as well as supportive care to prevent, control or treat complications and side effects).

\section{Outcomes}

This review will consider studies that include the following outcomes: 
150 COVID-19 infection outcomes:

151 a) Death

b) Invasive mechanical ventilation

c) Intensive care unit admission

d) Hospitalization

e) Severe and critical symptoms

\section{Types of studies}

This review will consider observational studies including prospective and retrospective cohort studies, case-control studies, analytical cross-sectional studies.

\section{Search strategy}

The search strategy will aim to locate both published and unpublished studies. The search strategy, including all identified keywords and index terms, it will be adapted for each included information source. The reference list of all studies selected for critical appraisal will be screened for additional studies. There will be no language restriction. We will include published and non-published studies from January/2020 to December/2021.

\section{Information sources}

Four general research strategies have been applied to the main electronic health databases: EMBASE (Elsevier, 1980-2021), Medline (PMC, 1966-2021), LILACS (by Virtual Health Library, 1982-2020) and The Cochrane Central Register of Controlled Trials (CENTRAL - Cochrane). The search strategies have contained descriptors and synonyms of hematologic neoplasm and COVID-19. There will be no language restriction. We will include published and non-published studies from January/2020 to December/2021. An initial limited search of PMC, EMBASE and Virtual Health Library (BVS) was undertaken to identify articles on the topic (see Additional File 2).

The following databases will also be searched for eligible studies: Trip Medical Database, SCOPUS, Web of Science, Cumulative Index to Nursing and Allied Health 
Literature (CINAHL), Australasian Medical Index, Chinese Biomedical Literature Database, British Library EThOS, RECAAP (Access Scientific Repository of Portugal), DART-Europe E-theses Portal, TROVE (National Library of Australia and local partner organizations). The grey literature will be searched through unpublished studies on <https://clinicaltrials.gov> website, the Brazilian Registry of Clinical Trials (ReBEC), PQCDT Dissertations \& Theses (ProQuest Dissertation \& Theses Global), WorldCat (World Catalog by Online Computer Library Center), the Digital Library of Theses and Dissertations of the University of São Paulo, Catalog of Theses \& Dissertations - CAPES, INCA (National Cancer Institute, Brazilian Ministry of Health) and abstracts in annals and lectures. Significant primary or secondary studies will be tracked to identify other possible eligible studies.

\section{Data Collection and analysis}

\section{Study selection}

All citations identified in research will be pooled and loaded into bibliographic software EndNote X8 version/2018. Duplicates references will be excluded. Three independent reviewers (POMH, NKH and ALM) will select the titles and abstracts for evaluation according to the inclusion criteria established for this review. The next step is the full text screening. Their citation details will be imported into the Joanna Briggs Institute System for the Unified Management, Assessment and Review of Information (JBI SUMARI) (Joanna Briggs Institute, Adelaide, Australia) [13]. The selected references will be retrieved in full, and their citation details imported into the Joanna Briggs Institute for Unified Management, Evaluation and Information Review System (JBI SUMARI) (Joanna Briggs Institute, Adelaide, Australia) [9]. The full text of the selected references will be evaluated according to the inclusion criteria. Texts that do not meet the inclusion criteria will be excluded from the review. The reasons for exclusion will be recorded and reported in the systematic review. In case of divergences between the reviewers during the stages of the study selection process, a fourth reviewer (VdSN-N) will be consulted. The results of the systematic review will be reported in full and presented in a flow chart of Preferred Reporting Items for Systematic Reviews and Metaanalyses (PRISMA) [14].

\section{Assessment of methodological quality}

Three independent reviewers (POMH, NKH and ALM) will critically assess the selected studies, as well as their methodological quality from the Joanna Briggs Institute's 
standardized critical assessment instruments for cohort, case-control and case series studies. Authors of papers will be contacted to request missing or additional data for clarification, where required. Any disagreements that arise will be resolved through discussion, or with a fourth reviewer. The results of critical appraisal will be reported in narrative form and in a table.

All studies, regardless of the results of their methodological quality, will undergo data extraction and synthesis (where possible). If possible, the results of critical appraisal will be incorporated into sensibility analysis on meta-analysis approach: type and duration of hematologic neoplasm and its treatments, staging of hematologic neoplasm, study design, number of patients and outcome results.

Any disagreements that arise between the reviewers will be resolved through discussion, or with a fourth reviewer. Authors of papers will be contacted to request missing or additional data where required.

\section{Data synthesis}

The results will be used in the meta-analysis when they are similar in at least two studies. The System for Unified Management, Evaluation and Review of Information (SUMARI), the main software of the Joanna Briggs Institute will be used for this meta-analysis. For dichotomous data, the relative risk will be calculated with $95 \%$ confidence intervals (CIs) as the estimated effect of the exposure. Continuous data will be expressed as mean and standard deviation and the differences between means with 95\% CIs will be used as an estimate of the intervention effect. We use mean adjusted difference for the studies that provide this data. A random-effects model will be used for the meta-analysis. If quantitative synthesis is not appropriate, a narrative synthesis will be provided. In case of including only case series studies, we will perform a proportional meta-analysis.

Inconsistencies between the results of the included studies will be ascertained by visual inspection of forest plots (no overlap of CIs around the effect estimates of the individual studies) and by Higgins or I 2 statistic, in which I $2>50 \%$ indicates a moderate probability of heterogeneity, and by chi2 test, where $\mathrm{p}<0.10$ indicates heterogeneity. ${ }^{15}$ The potential causes of heterogeneity between studies will be evaluated by subgroup analysis according to treatment type, hematologic malignancy subtype, neoplasm staging, race, geographic location, hospitalized and outcome patients. If the inconsistency was not explained by subgroup analysis, 
and more than 10 trials are included in the meta-analysis, a meta-regression using the metareg command available for the Stata statistical package will be performed (Stata Statistical Software 16 (Stata Statistical Software: Release 16. College Station, TX, StataCorp LLC, USA). For meta-regression we will use as categorical covariates (the risk of bias), gender, treatment type (wait-and-watch approaches, myeloablative chemotherapy or nonmyeloablative, target therapies, immunotherapy, radiotherapy and stem cell transplantation, as well as supportive care), hematologic malignancy's subtype (non-Hodgkin lymphomas, Hodgkin Disease, acute myeloid leukemia, acute lymphoid leukemia, chronic lymphoid leukemia, multiple myeloma, myelodysplastic syndrome, myeloproliferative diseases and chronic myeloid leukemia), neoplasm staging, race, geographic location. We will use as noncategorical variables: sample size, mean age of the participants.

\section{Assessing certainty in the findings}

For summary results from controlled studies the certainty of evidence will be followed the serie Grading of Recommendations, Assessment, Development and Evaluation (GRADE) for observational studies[15].

\section{Data extraction}

Data will be extracted from papers included in the review using the standardized data extraction tools in JBI SUMARI by two independent reviewers (POMH, NKH and ALM). The data extracted will include criteria relating to COVID-19 infection, underlying hematologic neoplasms diagnosis,

\section{Discussion}

Most of the current studies that discuss COVID and cancer include hematologic neoplasms as cancer, without distinguishing between their different categories of diagnosis, evolution and treatment. Hematologic neoplasms are a heterogeneous group of diseases consisting of acute or chronic leukemias, of the myeloid or lymphoid series; different degrees of differentiation from non-Hodgkin's lymphomas or Hodgkin's disease; multiple myeloma, myelodysplastic syndrome and myeloproliferative diseases such as polycythemia vera, primary myelofibrosis, essential thrombocythemia. All these categories of hematologic neoplasms have their peculiarities of treatment and prognosis that can not be generalized into a single category: cancer. We believe that not all hematologic neoplasms, as well as all types of treatment for 
these neoplasms, constitute a high risk for COVID-19. Therefore, our aim is to assess through a systematic review and meta-analysis whether all types of hematologic malignancies are a risk factor for severe COVID-19 infection.

This protocol will not limit the search to PMC and Embase as the two studies that preceded us. We will expand our search to Australian, Chinese, Portuguese, European and Brazilian scientific databases as described in the Information Sources section. We believe that research in these databases can contribute in an unprecedented way to the data in the literature, as many of these countries were the epicenter of the COVID-19 pandemic. Another difference between our protocol and previous studies is that we will be covering a longer period of time, including the second phase of the pandemic. Vijenthira et al reported that the risk of dying from COVID-19 may have been overestimated among patients with hematologic malignancy, as many of the included studies showed results from the early stages of the pandemic. It is possible that mortality rates will improve due to the increase in experience and therapeutic options and the improvement in the capacity of health systems to manage the flow of patients.

The accelerated pace in the generation of clinical data and publications on cancer patients and COVID-19 is remarkable. This represents a major challenge for health professionals and researchers in understanding the latest findings. Therefore, meta-analysis studies become essential to assess outcomes in larger cohorts of patients and trends in specific risk groups.

\section{List of abbreviation}

BVS: Virtual Health Library, Ministry of Health, Brazil.

CAPES: Coordination for Improvement of Higher Education, Brazil.

CI: Confidence Interval.

CINAHL: Cumulative Index to Nursing and Allied Health Literature.

COVID-19: Coronavirus disease of 2019.

CRD: Centre for Reviews and Dissemination.

DART-Europe: Digital Access to Research Theses -Europe E-theses Portal.

EMBASE: Excerpta Medica dataBASE.

EThOS: E-Theses Online Service provided by the British Library. IgG: Immunoglobulin G.

IgM: Immunoglobulin M.

INCA: Brazilian National Cancer Institute.

JBI: Joanna Briggs Institute, Adelaide, Australia. 
LDH: Lactate Dehydrogenase.

LILACS: Scientific health information from Latin America and the Caribbean countries.

IL-6: Inteleukin-6.

MEDLINE: Medical Literature Analysis and Retrieval System Online.

PMC: PubMed Central.

PQDT Dissertations \&Theses: ProQuest Dissertation \& Theses Global.

PRISMA: Preferred Reporting Items for Systematic Reviews and Meta-analyses. PRISMA-P: Preferred Reporting Items for Systematic Reviews and Meta-Analysis Protocols. PROSPERO: International Prospective Register of Systematic Reviews. PT: Prothrombin Time.

ReBEC: Brazilian Registry of Clinical Trials.

RECAAP: Access Scientific Repository of Portugal.

RT-PCR test: Reverse Transcription Polymerase Chain Reaction.

SARS-CoV-2: Severe Acute Respiratory Syndrome Coronavirus 2.

SCOPUS: Elsevier's abstract and citation database.

SUMARI: The System for Unified Management, Evaluation and Review of Information.

TROVE: Australian online libary database.

WHO: World Health Organization.

WorldCat: World Catalog by Online Computer Library Center.

\section{Declarations}

\section{Ethics approval and consent participate}

Not applicable.

\section{Consent for publication}

Not applicable.

\section{Competing interests}

The authors declare that they have no competing interests.

\section{Funding}

Not applicable.

\section{Authors' contributions}

POMH, NKH and VdSN-N conceptualised and design the study. POMH and VdSN-N drafted the manuscript protocol. POMH, NKH, ALM and VdSN-N critically revised the protocol and manuscript submitted. All authors read and approved the final manuscript.

\section{Acknowledgements}


The authors are grateful to Brazilian Centre for Evidence-based Healthcare: A JBI Centre of Excellence for reviewing this protocol.

\section{References}

1. Cazzola M. Introduction to a review series: the 2016 revision of the WHO classification of tumors of hematopoietic and lymphoid tissues. Blood. 2016; 127(20): 2361-2364.

2. Dhodapkar M, \& Dhodapkar K. (2015). Immune Modulation in Hematologic Malignancies. Semin Oncol. 2015; 42(4): 617-625.

3. Turner MR. Immune Modulation in Hematologic Malignancies. Physiology \& Behavior. 2016; 176(1):139-148.

4. Arber DA, Cousar JB. Hematopoietic Tumors: Principles of Pathologic Diagnosis. In: Greer JP, Foerster J, Rodgers GM, Paraskevas F, Glader B, Arber DA, \& Jr.Means RT. Clinical Hematology. $12^{\text {th }}$ ed. Philadelphia: Lippincott Williams \& Wilkins; 2009. 16631668

5. Meng Y, Lu W, Gguo E, \& Liu, J. Cancer history is an independent risk factor for mortality in hospitalized COVID-19 patients: a propensy score-matched analysis. J Hematol \& Oncol. 2020; 13(75):1-11.

6. Venkatesulu B P, Chandrasekar V T, Girdhar P, Advani P, Sharma A, Elumalai T, et al. A systematic review and meta-analysis of cancer patients affected by a novel coronavirus. JNCI Cancer Spectrum. 2021; 5 (2): pkaa102.

7. Vijenthira A, Gong I Y, Fox T A, Booth S, Cook G, Fattizzo B, et al. Outcomes of patients with hematologic malignancies and COVID-19: a systematic review and metaanalysis of 3377 patients. Blood. 2020; 136 (25): 2881-2892.

8. Kim JS, Lee KH, Kim GE, Kim S, Yang JW, Li H, et al. Clinical characteristics and mortality of patients with hematologic malignancies and COVID-19: a systematic review. Eur Rev Med Pharmacol Sci. 2020; 24(22): 11926-11933.

9. Wang Q, Berger NA, Xu R. When hematologic malignancies meet COVID-19 in the United States: Infections, death and disparities. Blood Rev. 2021; 47: 100775. 
10. Carpenter C R, Mudd P A, West C P, Wilber E, Wilber S T. Diagnosing COVID-19 in the Emergency Department: A Scoping Review of Clinical Examinations, Laboratory Tests, Imaging Accuracy, and Biases. Acad Emerg Med. 2020;27(8):653-670.

11. Bruneau J., Molina T.J. WHO Classification of Tumors of Hematopoietic and Lymphoid Tissues. $5^{\text {th }}$ rev. ed. Molina T.J. (eds) Hematopathology. Encyclopedia of Pathology. Berlim: Springer; 2020. 368p.

12. Moola S, Munn Z, Tufanaru C, Aromataris E, Sears K, Sfetcu R, et al. Chapter 7: Systematic reviews of etiology and risk. In: Aromataris E, Munn Z (Editors). Joanna Briggs Institute Reviewer's Manual. The Joanna Briggs Institute, 2017. Available from https://reviewersmanual.joannabriggs.org/

13. Aromataris E, Munn Z (Editors). The Joanna Briggs Institute. Joanna Briggs Institute Reviewer's Manual. 2017. Available from https://reviewersmanual.joannabriggs.org/ 14. Moher D, Liberati A, Tetzlaff J, Altman DG; PRISMA Group. Preferred reporting items for systematic reviews and meta-analyses: the PRISMA statement. PLoS Med. 2009; 6(7):e1000097.

15. Guyatt GH, Oxman AD, Sultan S, Glasziou P, Akl EA, Alonso-Coello P, et al. GRADE guidelines: 9. Rating up the quality of evidence. J Clin Epidemiol. 2011; 64(12):1311-1316. 


\section{Supplementary Files}

This is a list of supplementary files associated with this preprint. Click to download.

- Additionalfile1.pdf

- Additionalfile2.pdf 